\title{
Rendezvous therapy with endoscopic and endovascular treatments for rectal arteriovenous malformation: A case report
}

An 84-year-old man was admitted to our hospital with recurring hematochezia. Six months earlier, he had undergone transcatheter arterial embolization using platinum coils for hemorrhagic rectal arteriovenous malformation.

Contrast-enhanced computed tomography $(\mathrm{CT})$ revealed a recurrence of the rectal arteriovenous malformation ( Fig. 1), and colonoscopy revealed erosion with pulsation in the lower rectum ( Fig.2). The diagnosis was rectal arteriovenous malformation rebleeding, which is typically treated using transcatheter arterial embolization and surgical resection [1-3]. However, transcatheter arterial embolization alone does not eliminate the abnormal vessels, and surgical resection was considered too invasive because of the patient's advanced age. Therefore, a less invasive curative therapy was attempted to control bleeding from the malformation using a combination of endoscopic injection sclerotherapy with n-butyl cyanoacrylate-lipiodol and endovascular therapy with balloon catheters ( Video 1).

A 1.9-Fr catheter was initially advanced to the inferior mesenteric artery via a percutaneous approach, and arteriography revealed typical arteriovenous malformation findings (abnormal vessels called a "nidus" in the arterial phase and early venous return in the capillary phase). A 2.7-Fr balloon catheter was then advanced to the superior rectal vein via a percutaneous transhepatic approach to occlude it and prevent the migration of n-butyl cyanoacrylate-lipiodol into the portal vein. Colonoscopy subsequently revealed spurting bleeding from the rectal erosion. To control the hemorrhage, the 1.9-Fr catheter was exchanged for a 2.7-Fr balloon catheter, and balloon occlusion of the inferior mesenteric artery resulted in temporary hemostasis. We injected $3 \mathrm{~mL}$ of $50 \%$ n-butyl cyanoacry-

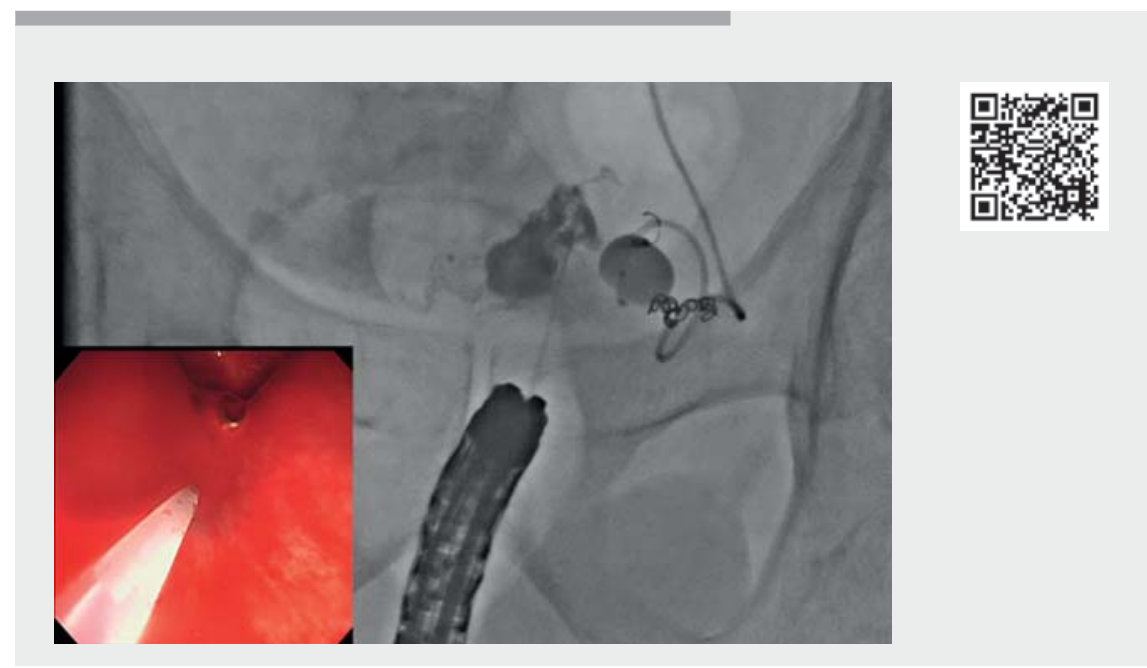

Video 1 A combination of endoscopic injection sclerotherapy with n-butyl cyanoacrylate-lipiodol mixture and endovascular therapy with balloon catheters for bleeding from a rectal arteriovenous malformation.

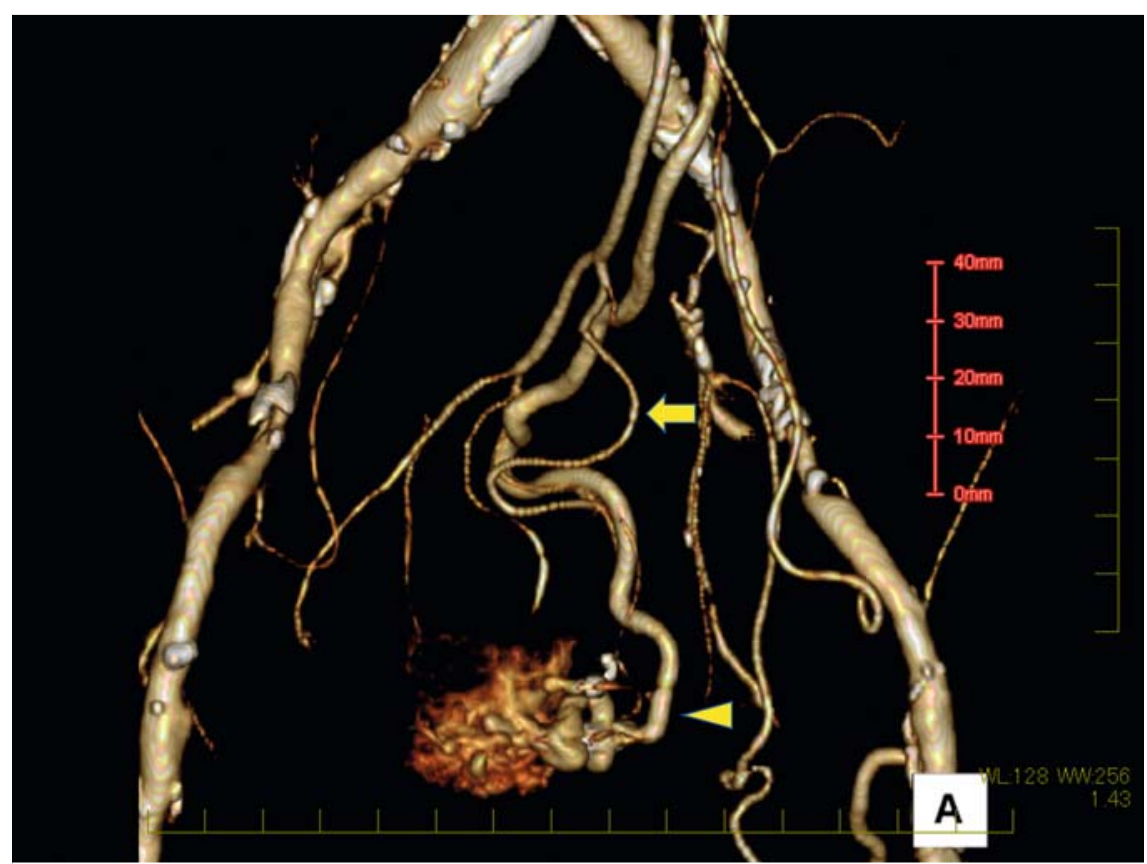

- Fig. 1 Three-dimensional reconstruction of pre-treatment contrast-enhanced computed tomography findings, which revealed recurrence of the rectal arteriovenous malformation. The feeding artery was considered the superior rectal artery (arrow) and the draining vein was considered the superior rectal vein (arrowhead). 


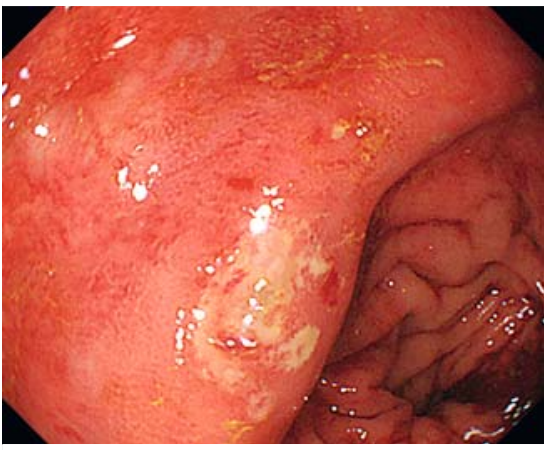

Fig. 2 Pre-treatment colonoscopy revealed erosion with pulsation in the lower rectum, which was considered the bleeding point.

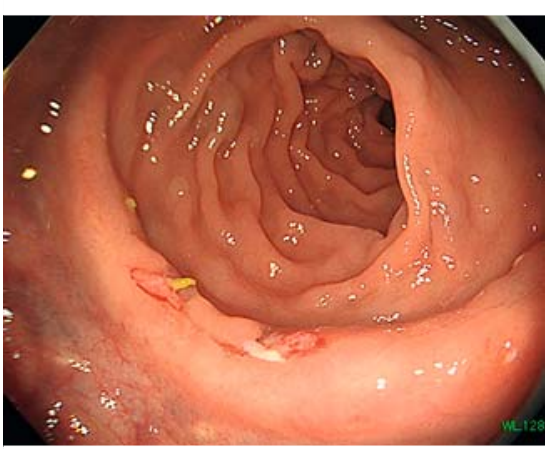

- Fig. 4 Post-treatment colonoscopy at one month revealed minor artificial erosion without pulsation in the lower rectum.

late-lipiodol using a 23-G needle directly at the bleeding point under balloon flow control of both the superior rectal vein and inferior mesenteric artery, and we observed successful n-butyl cyanoacrylate-lipiodol accumulation in the nidus under fluoroscopy.

Follow-up contrast-enhanced CT 1 month later revealed the disappearance of the nidus and a dilated vein draining the arteriovenous malformation ( $>$ Fig.3); colonoscopy revealed only minor artificial erosion without pulsation ( $\triangleright$ Fig. 4 ).

Endoscopy_UCTN_Code_TTT_1AT_2AF

\section{Competing interests}

The authors declare that they have no conflict of interest.

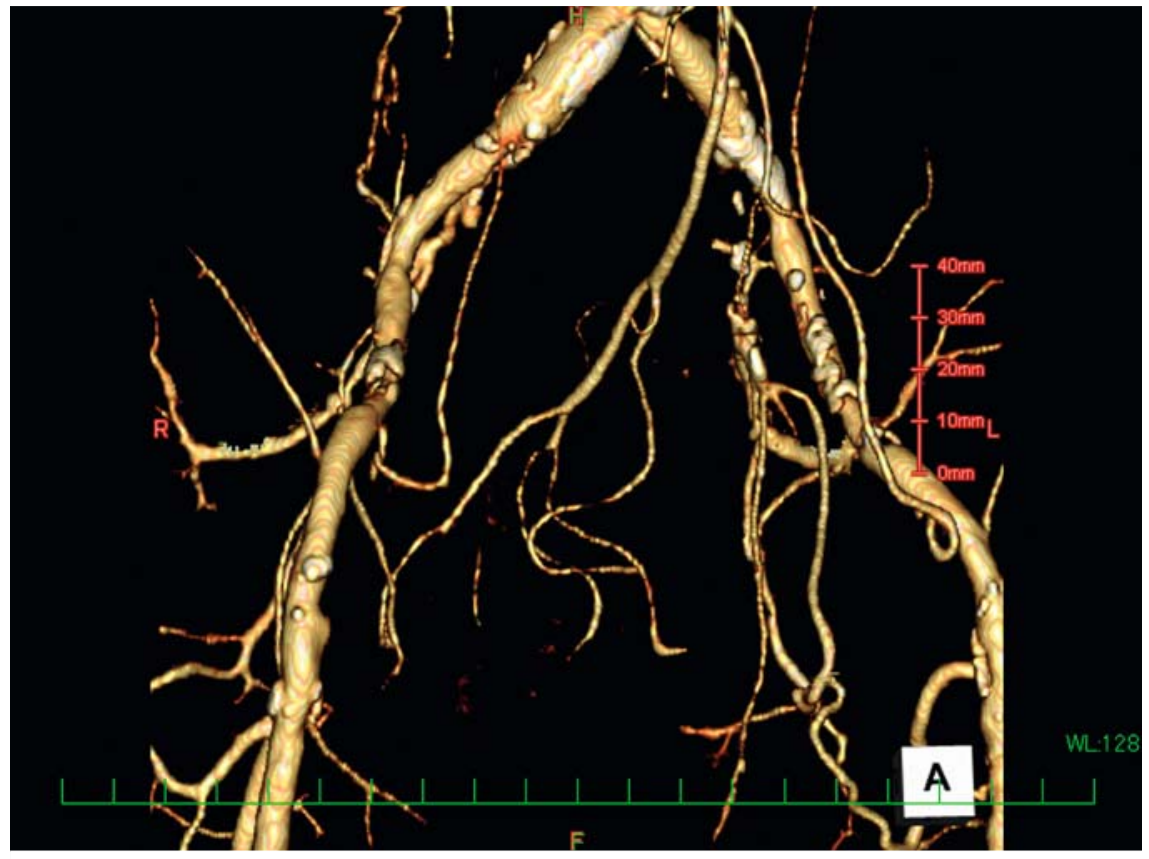

- Fig. 3 Three-dimensional reconstruction of post-treatment contrast-enhanced computed tomography findings. The one-month follow-up revealed no findings of arteriovenous malformation, with disappearance of the abnormal vessels and dilated draining vein.

The authors

Takashi Matsuda', Tsuyoshi Ishikawa², Takashi Oono $^{2}$, Ryo Sasaki², Isao Hidaka², Munemasa Okada ${ }^{3}$, Isao Sakaida ${ }^{1,2}$

1 Department of Liver Regenerative Medicine, Yamaguchi University Graduate School of Medicine, Ube, Japan

2 Department of Gastroenterology and Hepatology, Yamaguchi University Graduate School of Medicine, Ube, Japan

3 Department of Radiology, Yamaguchi University Graduate School of Medicine, Ube, Japan

\section{Corresponding author}

\section{Takashi Matsuda, MD}

Department of Liver Regenerative Medicine, Yamaguchi University Graduate School of Medicine, 1-1-1 Minami-Kogushi, Ube, Yamaguchi 7558505, Japan Fax: +81-836-22-2240

t.mazda@yamaguchi-u.ac.jp

\section{References}

[1] Mallios A, Laurian C, Houbballah R et al. Curative treatment of pelvic arteriovenous malformation - an alternative strategy: transvenous intra-operative embolisation. Eur J Vasc Endovasc Surg 2011; 41: 548-553

[2] Ishikawa S, Mukai S, Hirata Y et al. Rectal arteriovenous malformation treated by transcatheter arterial embolization. Case Rep Gastroenterology 2020; 14: 7-14

[3] Hayakawa H, Kusagawa M, Takahashi $\mathrm{H}$ et al. Arteriovenous malformation of the rectum: report of a case. Surg Today 1998; 28: $1182-$ 1187

\section{Bibliography}

Endoscopy 2022; 54: E77-E78

DOI 10.1055/a-1388-6021

ISSN 0013-726X

published online 15.3.2021

(c) 2021. Thieme. All rights reserved.

Georg Thieme Verlag KG, Rüdigerstraße 14, 70469 Stuttgart, Germany 